Revista Brasileira de Agricultura Irrigada v.13, nº.5, p. 3662 - 3675, 2019

ISSN 1982-7679 (On-line)

Fortaleza, CE, INOVAGRI - http://www.inovagri.org.br

DOI: $10.7127 /$ rbai.v13n5001128

Protocolo 1128.19 - 26/03/2020 Aprovado em 04/04/2020

\title{
ESTIMATIVA DA PRODUTIVIDADE PRIMÁRIA BRUTA DA VEGETAÇÃO NATIVA E CULTIVO IRRIGADO ATRAVÉS DE SENSORIAMENTO REMOTO
}

\author{
Antônio Erivando Bezerra ${ }^{1}$, Francisco Linco de Souza Tomaz ${ }^{2}$, Johny de Souza Silva ${ }^{3}$, Carlos \\ Wagner Oliveira $^{4}$, João Miguel de Moraes Neto ${ }^{5}$
}

\begin{abstract}
RESUMO
A cultura da bananeira (Musa spp.) é de extrema importância para a região semiárida do Brasil. No entanto, fatores edafoclimáticos, como a seca, contribuem para a redução da produtividade. Algumas técnicas de monitoramento através do sensoriamento remoto vêm auxiliando produtores no manejo da propriedade. Objetivou-se com este trabalho, estimar a Produtividade Primaria Bruta (PPB) para vegetação nativa e cultivo irrigado da bananeira, na região do Cariri Cearense, através de sensoriamento remoto. $\mathrm{O}$ experimento foi realizado em área localizada na região sul do estado do Ceará que contêm unidades produtoras de banana irrigada, denominadas de sítios 1, 2 e 3, e uma área de vegetação nativa da caatinga. As avaliações foram realizadas em cinco datas distintas. As imagens foram geradas pelos sensores OLI/TIRS do satélite Landsat 8, órbita 217 e ponto 65. A determinação da PPB foi realizada a partir da compilação dos modelos da radiação fotossinteticamente ativa. Verificou-se que o déficit hídrico reduziu a PPB na área de vegetação nativa, entretanto, observou-se pouca ou nenhuma redução na área irrigada. A deficiência hídrica, portanto, afetou diretamente a PPB, comprometendo a atividade fotossintética e consequentemente a produtividade da cultura.
\end{abstract}

Palavras-Chave: musa spp., landsat, uso e ocupação do solo.

\section{ESTIMATION OF GROSS PRIMARY PRODUCTIVITY OF NATIVE VEGETATION AND IRRIGATED CULTIVATION THROUGH REMOTE SENSING}

\footnotetext{
ABSTRACT

The banana crop (Musa spp.) is extremely important for the semiarid region of Brazil. However, edaphoclimatic factors, such as drought, contribute to reduced productivity. Some remote sensing monitoring techniques have been assisting producers in managing the property. The aim of this work

${ }^{1}$ Mestre em Engenharia Agrícola, Depto. de Engenharia Agrícola, Universidade Federal de Campina Grande, UFCG, Campina Grande - PB, aerivando86@gmail.com.

${ }^{2}$ Mestrando em Fitotecnia, UFC, Fortaleza-CE, e-mail: chicolinco@ hotmail.com

${ }^{3}$ Doutorando em Fitotecnia, UFC, Fortaleza-CE, e-mail:johny.ufca@gmail.com

${ }^{4}$ PhD em Biosystems Engineering, Professor da UFCA, Crato-CE, e-mail: carlos.oliveira@ufca.edu.br

${ }^{5}$ Doutor em Recursos Naturais, Professor da UFCG, Campina Grande-PB, e-mail: moraes@ deag.ufcg.edu.br
} 
was to estimate the Gross Primary Productivity (GPP) for native vegetation and irrigated banana cultivation, in the Cariri Cearense region, by remote sensing. The experiment was carried out in an area located in the southern region of Ceará State that contains, and three irrigated banana producing units, called sites 1, 2 and 3, an area of native caatinga vegetation. Evaluations were performed on five different dates. The images were generated by the Landsat 8 satellite OLI / TIRS sensors, orbit 217 and point 65. The determination of the GPP was performed by compiling the models of photosynthetically active radiation. Water deficit reduced GPP in native vegetation area, however, there was little or no reduction in irrigated area. Water deficiency, therefore, directly affected GPP, compromising photosynthetic activity and consequently crop yield.

Keywords: musa spp., landsat, land use and occupation.

\section{INTRODUÇÃO}

A bananeira (Musa spp.) é uma das culturas mais importantes para a alimentação humana. Em 2017, a produção global foi de quase 202 milhões de toneladas (FAO Stat, 2019). No Brasil, os dados mais recentes, referentes à safra de 2017, mostram o país na quinta posição mundial na produção de banana, com uma produção de 7,13 milhões de toneladas, colhidas em 533.323 hectares e produtividade média em torno de $13,49 \mathrm{t} \mathrm{ha}^{-1}$ (IBGE, 2017).

$\mathrm{O}$ sequestro de carbono realizado pelos ecossistemas agrícolas e florestais, no contexto das alterações climáticas, é essencial para mitigar os danos e suas consequências (SHARMA et al., 2013). O principal processo envolvendo a contenção de carbono pelos ecossistemas é chamado de Produtividade Primária Bruta - PPB (Gross Primary Productivity), que ocorre a partir da conversão de energia luminosa em fitomassa. A PPB refere-se, portanto, ao processo denominado de fotossíntese, que constitui um dos principais processos que controlam a troca de dióxido de carbono $\left(\mathrm{CO}_{2}\right)$ entre a biosfera e a atmosfera, sendo ferramenta bastante relevante para compensar as emissões de $\mathrm{CO}_{2}$ (ALMEIDA et al., 2018).

Existem diversos métodos destinados à quantificação do carbono estocado na vegetação, dentre os quais se destaca o da covariância de vórtices turbulentos (BEER et al., 2010; CABRAL et al., 2011). Apesar de sua relevância na quantificação do $\mathrm{CO}_{2}$, seu custo e operacionalidade muitas vezes se apresentam restritivos para vários grupos de pesquisadores nos países em desenvolvimento, além disso, sua representatividade cobre apenas alguns quilômetros quadrados (SILVA et al., 2013).

Tal fato contribui para o crescimento da aplicação do sensoriamento remoto utilizando imagens de satélite, com ampla cobertura espacial, baixo custo e maior operacionalidade. Muitos modelos da PPB baseados em sensoriamento remoto se fundamentam na relação entre eficiência de uso da luz $(\varepsilon)$, fração da radiação fotossinteticamente ativa absorvida (APAR) e radiação fotossinteticamente ativa (RFA) (ALMEIDA et al., 2018).

$\mathrm{Na}$ região semiárida, apesar dos fatores climáticos, energia disponível e temperatura favorecerem o desenvolvimento da bananeira, a irregularidade de distribuição das chuvas e o manejo irracional da irrigação concorrem para reduzir sua produtividade (COSTA et al., 2012). O consumo de água pela bananeira é elevado e constante, sendo que para expressar todo seu potencial produtivo, a cultura necessita de $2500 \mathrm{~mm}$ de água por ano (ROBINSON; SAÚCO, 2010).

Diante disso, objetivou-se com este trabalho, estimar os efeitos do déficit hídrico sobre a produtividade primaria bruta para vegetação nativa e cultivo irrigado da bananeira, na região do Cariri Cearense, através de sensoriamento remoto.

\section{MATERIAL E MÉTODOS}

Este trabalho foi realizado entre os municípios de Barbalha e Missão Velha, 
localizados na região sul do estado do Ceará, possuindo as seguintes coordenadas geográficas: $7^{\circ} 19^{\prime}$ de latitude $\operatorname{Sul}, 39^{\circ} 11^{\prime}$ de longitude Oeste e elevação de 403 metros em relação ao nível do mar (Figura 1). Segundo classificação de Koppen-Geiger, o clima da região é do tipo quente e úmido (Aw), com regime de chuvas tropical austral (w) totalizando $1.075,8 \mathrm{~mm}$ de precipitação média anual, temperatura média anual de $24,9{ }^{\circ} \mathrm{C}$, umidade relativa média entre 40 e $60 \%$, taxa de insolação de 2.848 horas anuais, velocidade média do vento de $1,90 \mathrm{~m} \mathrm{~s}^{-1} \mathrm{e}$ taxa evaporativa superior a $2000 \mathrm{~mm} \mathrm{ano}^{-1}$ (LIMA et al., 2017).

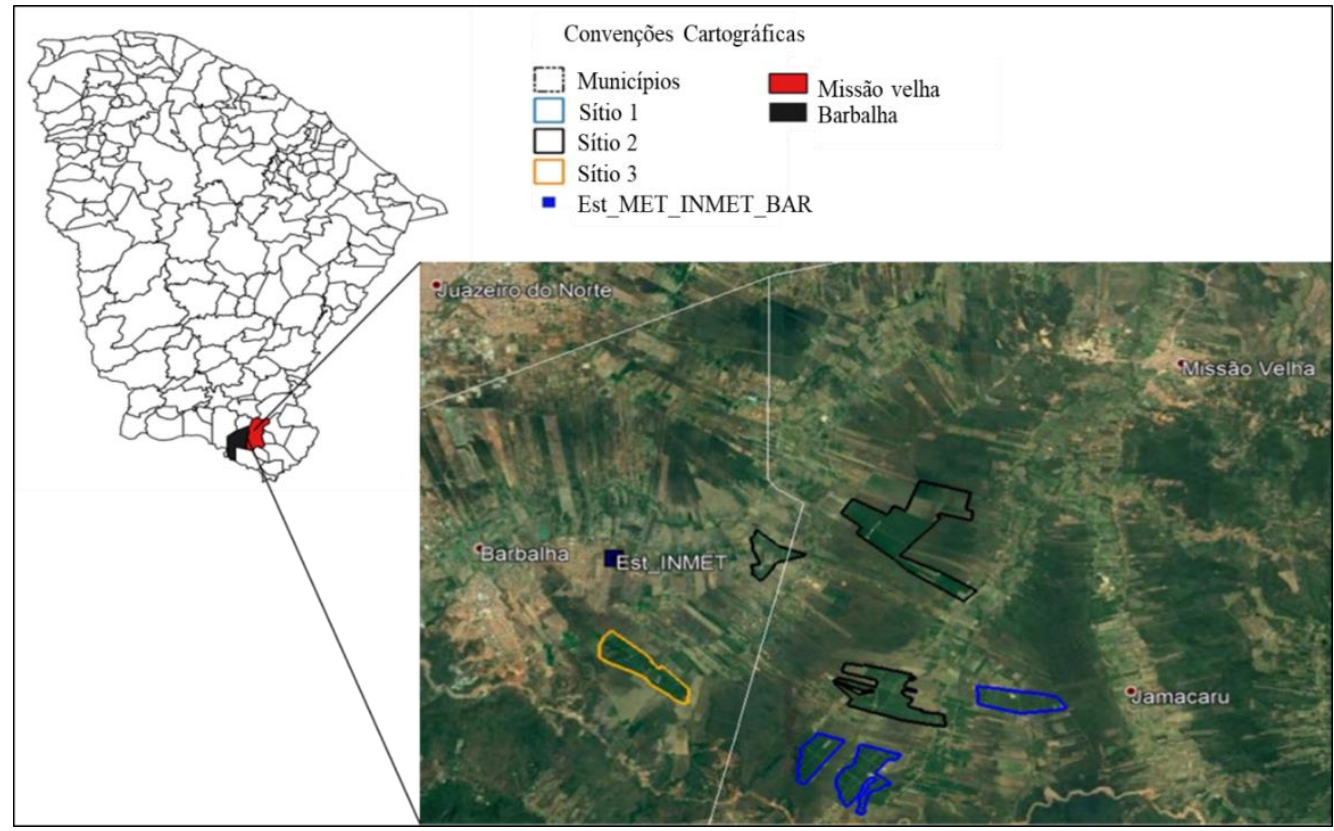

Figura 1. Mapa de Localização da área de interesse para realização da pesquisa.

O cenário utilizado para realização da pesquisa possui área superior a $10 \mathrm{~km}^{2}$, no mesmo, foram selecionadas três parcelas experimentais constituídas por três unidades produtoras de banana irrigada, as quais foram denominadas de sítios 1, 2 e 3. Em todas as unidades de produção, foram identificadas áreas com os seguintes tempos de cultivo: bananeira recém-implantada, bananeira cultivada há quatro anos, cultivo estabelecido entre quatro e dez anos e, por fim, cultivo estabelecido entre dez e vinte anos. Além do cultivo irrigado, foi identificado remanescente da vegetação nativa da caatinga, pastagens e cultivo de sequeiro.

Foram obtidas imagens geradas pelos sensores OLI/TIRS do satélite Landsat 8, órbita 217 e ponto 65, junto ao USGS (United States Geological Survey) na plataforma "Earth Explorer", para as seguintes datas: 3 de março, 22 de maio, 9 de julho, 29 de outubro e
14 de novembro de 2016, escolhidas devido apresentarem melhores qualidade de processamento, tendo em vista a menor cobertura de nuvens sob o local estudado. Para posterior obtenção dos elementos do balanço energético na superfície, foram utilizados dados tabulares da plataforma de coleta de dados meteorológicos automatizados, tais como: temperatura do ar, velocidade do vento, temperatura do ponto de orvalho, pressão atmosférica, balanço de radiação e precipitação. Os referidos dados foram também utilizados nos processos de validação dos modelos e no cálculo da evapotranspiração de referência (ETr), através do software RefET (ALLEN, 2010).

Para a calibração das imagens e processamento dos modelos, foram realizadas composições com sete bandas espectrais (bandas 2, 3, 4, 5, 7 e 10). Além disso, fez-se necessário a identificação de alguns 
parâmetros de ajuste utilizados no cômputo dos diferentes componentes dos balanços de radiação e energia, tais como: Ângulo de Elevação Solar - E (degree), Cosseno do Ângulo Zenital - Cos Z $\left(^{\circ}\right)$, Temperatura do $\mathrm{Ar}$ - Ta $\left({ }^{\circ} \mathrm{C}\right)$, Umidade Relativa do Ar - URA $(\%)$, Pressão Atmosférica - Po $(\mathrm{kPa})$,

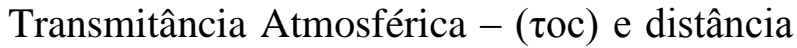
média entre a Terra e o Sol - dr (unidade astronômica). O processamento dos modelos foi realizado no software ArcGIS, versão 10.2 e os mapas foram confeccionados através do software Qgis, versão 2.18.

Para analisar o grau de influência dos fatores edafoclimáticos sobre a produtividade primária bruta do cultivo irrigado da banana e da vegetação nativa, foram confeccionados mapas de uso e ocupação do solo, com base na técnica de classificação supervisionada. Essa técnica permite correlacionar os valores espectrais dos pixels com as classes de cobertura no solo, de modo que o usuário indique várias regiões com características espectrais conhecidas e o algoritmo escolhido realiza a extrapolação e a classificação do restante da imagem (ZHONG et al., 2016).

A produtividade primária bruta (PPB) corresponde à fotossíntese bruta, usada para mensurar a quantidade total de energia ou carbono produzida pela planta em um período de tempo, através da fotossíntese (ALMEIDA et al., 2018). Sua determinação pode ser realizada através da equação (1), que consiste na compilação dos modelos de estimativa da radiação fotossinteticamente ativa absorvida pela vegetação (APAR), proposto por Monteith (1972), e da eficiência do uso da luz ( $\varepsilon$ ), de Field et al. (1995), adaptado por Bastiaanssen e Ali (2003), que sugerem o emprego da fração evaporativa (w) como elemento quantificador do status da água pela vegetação (DOUGHTY et al., 2018).

$\mathrm{PPB}=\varepsilon \times \sum(\operatorname{APAR}(\mathrm{t}) \mathrm{t})$

Em que: PPB é a Produtividade Primária Bruta $\left(\mathrm{kg} \mathrm{m}^{-2}\right) ; \varepsilon$ é a eficiência do uso da luz $\left(\mathrm{g} \mathrm{MJ}^{-}\right.$ ${ }^{1}$ ); APAR é a Radiação Fotossinteticamente Ativa Absorvida ( $\mathrm{W} \mathrm{m}^{-2}$ ); t é o tempo (dias).
A APAR foi calculada a partir da equação (2), segundo Bastiaanssen e Ali (2003):

$\mathrm{APAR}=\mathrm{f} \times \mathrm{PAR}$

Em que: $f$ é a fração da radiação fotossinteticamente ativa, interceptada pela área foliar da planta. Segundo Bastiaanssen e Ali (2003), a f pode ser obtida pela equação (3):

$F=(-0,161+1,257 \times$ NDVI $)$

O Índice de Vegetação por Diferença Normalizada (NDVI) indica a quantidade e a condição de massa verde na superfície da vegetação, sendo estimado pela equação (4) (ALLEN et al., 2010). Geralmente, os valores do NDVI variam de -1 a +1 . No entanto, em superfícies vegetadas, os valores são sempre positivos, indo de $0 \mathrm{a}+1$, sendo negativos em nuvens e espelhos d'água.

$N D V I=\frac{(\rho 5-\rho 4)}{(\rho 5+\rho 4)}$

Em que: $\rho 5$ é a refletividade da banda 5 (infravermelho próximo); $\rho 4$ é a refletividade da banda 4 (vermelho).

A PAR corresponde a radiação fotossinteticamente ativa de grande escala (W $\mathrm{m}^{-2}$ ), calculada através da equação (5):

PAR $=$ a x Rs_24h

Em que: a é a porcentagem da Rs_24h referente à radiação fotossinteticamente ativa, usada para ativação da clorofila foliar no processo de fotossíntese. A eficiência do uso da luz $(\varepsilon)$, também denominada eficiência real do uso da luz, expressa em $\mathrm{g} \mathrm{C} \mathrm{MJ}^{-1}$, varia ao longo da estação de crescimento da planta, portanto, será menor do que o valor $\varepsilon$ máximo. Embora dependa de outros elementos da comunidade vegetal, pode ser quantificada pelo modelo de Field et al. (1995), modificado por Bastiaanssen e Ali (2003), através da equação (6): 
$\varepsilon=\varepsilon^{\prime} \times \mathrm{T} 1 \times \mathrm{T} 2 \times \mathrm{W}$

Em que: $\varepsilon$ 'é o fator de conversão máximo da luz para produção de biomassa acima do solo quando as condições ambientais são ótimas, no caso do cultivo da banana, classificada como C3.

$\mathrm{T}_{1}=0,8+0,02 \mathrm{~T}_{\mathrm{opt}}-0,0005 \mathrm{~T}_{\mathrm{opt}}^{2}$

$\mathrm{T}_{2}=\frac{1}{1+\exp \left(0,2 \mathrm{~T}_{\mathrm{opt}}-10-\mathrm{T}_{\mathrm{dia}}\right)} \times \frac{1}{1+\exp \left[0,3\left(-\mathrm{T}_{\mathrm{opt}}-10+\mathrm{T}_{\mathrm{dia}}\right)\right]}$

Em que: $\mathrm{T}_{\mathrm{opt}}$ é a temperatura média do ar $\left({ }^{\circ} \mathrm{C}\right)$ do mês de máximo NDVI; $\mathrm{T}_{\text {dia }}$ é Temperatura média diária do ar $\left({ }^{\circ} \mathrm{C}\right)$.

A fração evaporativa (W) representa a contribuição da água na fotossíntese e pode ser determinada com base no balanço de energia, segundo Bastiaanssen e Ali. (2003) pela equação (9). A determinação dos elementos do balanço de energia na superfície foi realizada através do algoritmo METRIC, que segundo Allen et al. (2010) consiste em colocar o fluxo de calor latente (LE) em evidência e realizar a simples diferença entre o saldo de radiação $(\mathrm{Rn})$, o fluxo de calor no solo (Go) e o fluxo de calor sensível $(\mathrm{H})$, conforme a equação (10).

$\mathrm{W}=\Lambda=\frac{\mathrm{LE}}{(\mathrm{Rn}-\mathrm{Go})}$

$\mathrm{LE}=\mathrm{Rn}-\mathrm{Go}-\mathrm{H}$

Em que: Rn é o saldo de radiação $\left(\mathrm{Wm}^{-2}\right) ; \mathrm{G}$ é o fluxo de calor para o solo $\left(\mathrm{Wm}^{-2}\right)$; $\mathrm{H}$ é o fluxo de calor sensível $\left(\mathrm{Wm}^{-2}\right)$; LE é o fluxo
Considerou-se o valor de 2,5 (g $\left.\mathrm{MJ}^{-1}\right)$, indicado por Bastiaanssen e Ali, (2003), para a maioria das plantas (C3 e $\mathrm{C} 4)$.

Os termos $\mathrm{T}_{1}$ e $\mathrm{T}_{2}$ da equação 6 refletem a contribuição da temperatura na eficiência de uso da luz pelas plantas, podendo ser calculados pelas equações 7 e 8 .

de calor latente $\left(\mathrm{Wm}^{-2}\right)$. Visando avaliar as possíveis variações nos valores da PPB do cultivo irrigado da bananeira, realizou-se um experimento em delineamento inteiramente casualizado, em esquema fatorial $3 \times 3$ (três unidades produtoras e três tempos de cultivo), com 5 repetições (avaliações espaçadas no tempo: 3 de março, 22 de maio, 9 de julho, 29 de outubro e 14 de novembro de 2016).

As variáveis foram submetidas à análise de variância pelo teste $\mathrm{F}$ e quando significativas, foram submetidas ao teste de Tukey a $5 \%$ de probabilidade, utilizando-se o software estatístico SISVAR versão 5.6 (FERREIRA, 2011).

\section{RESULTADOS E DISCUSSÃO}

O resultado da análise de variância da variável PPB está apresentado na Tabela 1.

Os resultados indicam efeito significativo tanto nos fatores isolados, quanto na interação, em todas as datas avaliadas. 
Tabela1. Resumo da análise de variância para a variável PPB observada em vegetação nativa e cultivo irrigado da bananeira, na região do Cariri Cearense.

\begin{tabular}{ccccccc}
\hline \multirow{2}{*}{ FV } & \multirow{2}{*}{ GL } & \multicolumn{5}{c}{ Quadrado Médio } \\
\cline { 3 - 7 } & & $03 / 03 / 2016$ & $22 / 05 / 2016$ & $09 / 07 / 2016$ & $29 / 10 / 2016$ & $14 / 11 / 2016$ \\
\hline Sítio (A) & 2 & $167,49 * *$ & $112,42^{* *}$ & $144,34 * *$ & $1026,52^{* *}$ & $563,69 * *$ \\
Tempo (B) & 2 & $298,12^{* *}$ & $646,27^{* *}$ & $1385,01^{* *}$ & $8090,85^{* *}$ & $2895,03 * *$ \\
A x B & 4 & $33,57^{* *}$ & $20,36^{* *}$ & $29,36^{* *}$ & $35,16^{* *}$ & $51,86^{* *}$ \\
Resíduo & 11185 & 0,44 & 0,22 & 0,41 & 1,41 & 0,69 \\
\hline Média & --- & 6,97 & 7,52 & 6,75 & 7,08 & 5,29 \\
CV (\%) & --- & 9,55 & 6,22 & 9,53 & 16,80 & 15,65 \\
\hline
\end{tabular}

**significativo a 5\% de significância pelo teste $\mathrm{F}$

Na Figura 2, se observa valores médios mensais para temperaturas máxima e mínima e o acumulado mensal da precipitação durante o período de avaliação.

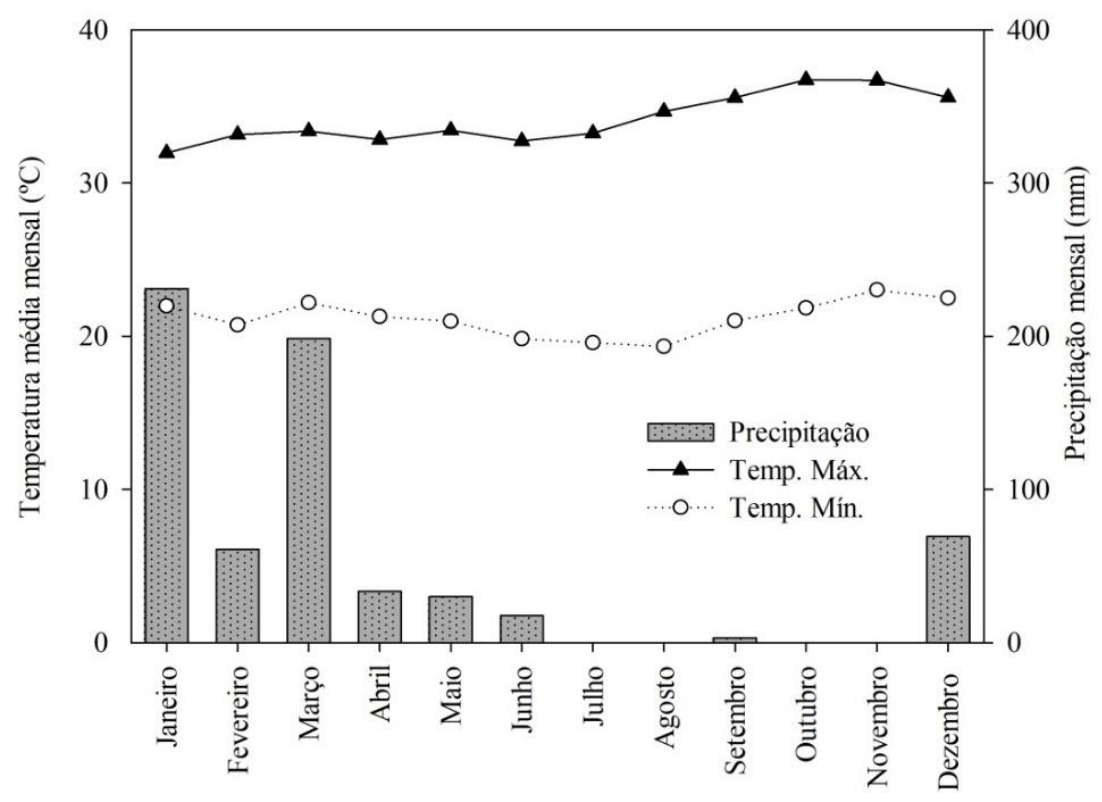

Figura 2. Dados climáticos do município de Barbalha, Ceará, no ano de 2016.

Com base nos dados meteorológicos analisados, pode-se inferir que os meses mais quentes foram outubro e novembro, com temperatura máxima média em torno de $35^{\circ} \mathrm{C}$, enquanto que nos meses de julho e agosto, observam-se baixa temperatura média mínima, com valores em torno de $20{ }^{\circ} \mathrm{C}$. Em relação à precipitação, houve predominância de chuvas no início do ano, com destaque para os meses de janeiro, fevereiro e março, com variações entre oitenta e superior a duzentos milímetros, corroborando com a literatura consultada, uma vez que Medeiros et al. (2013), confirmou a predominância de déficit hídrico na região metropolitana do Cariri cearense durante maioria dos meses do ano. Através da técnica da classificação supervisionada, foram confeccionados mapas de uso e ocupação do solo (Figura 3).

A vegetação no entorno das parcelas amostrais foi dividida em três classes, denominadas como vegetação densa com exemplares arbustivos, semidensa com exemplares arbóreos, e rala, contemplando a pastagem nativa ou cultivada, constituída principalmente por gramíneas e plantas rasteiras, corroborando com Brandão et al. (2012). 
ESTIMATIVA DA PRODUTIVIDADE PRIMÁRIA BRUTA DA VEGETAÇÃO NATIVA E CULTIVO IRRIGADO ATRAVÉS DE SENSORIAMENTO REMOTO
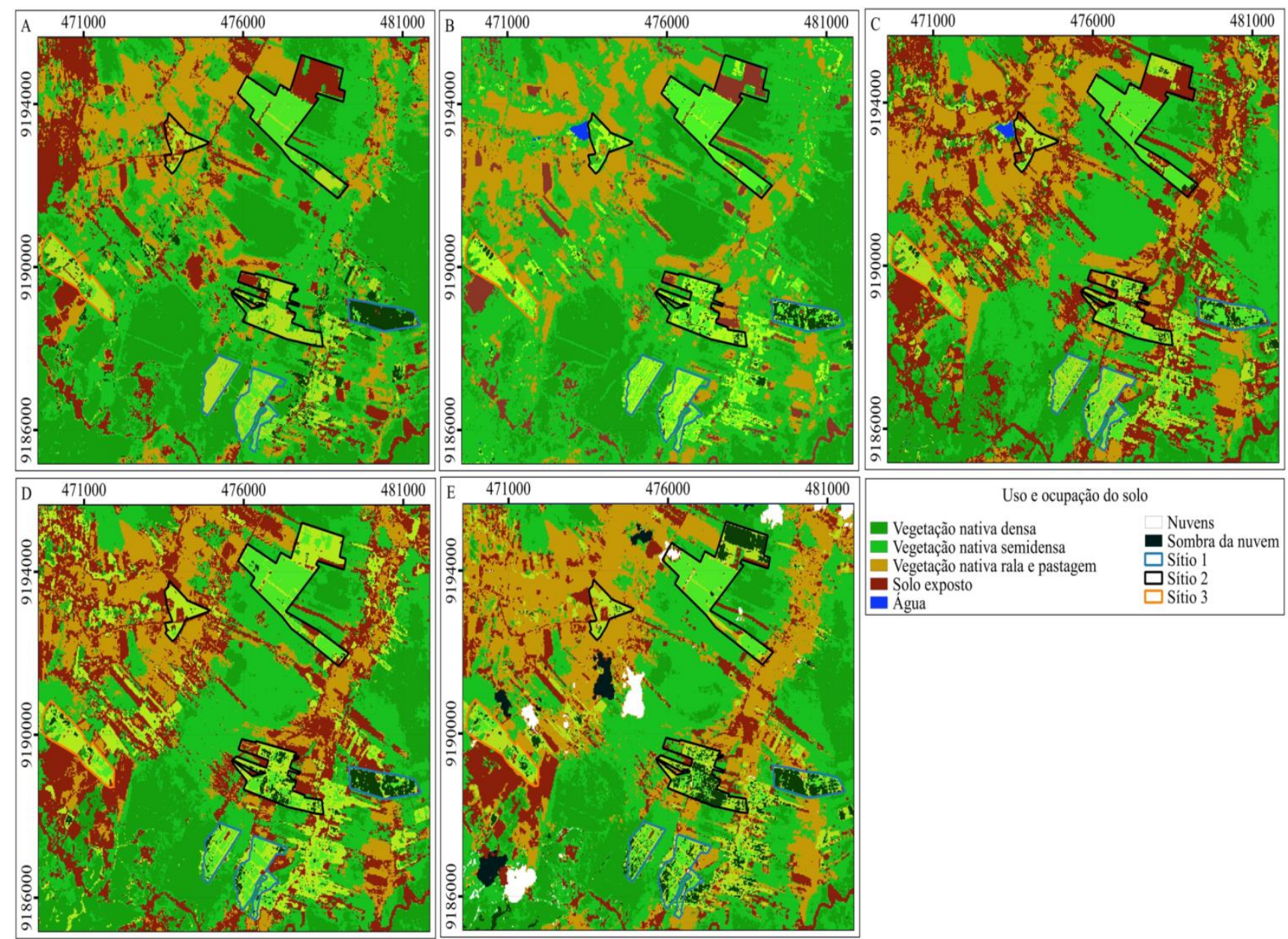

Figura 3. Espacialização geográfica das classes de uso e ocupação do solo para a área em estudo no decorrer das datas: 3 de março (A), 22 de maio (B), 9 de julho (C), 29 de outubro (D) e 14 de novembro (E), do ano de 2016. 
O cultivo irrigado da banana destacado em polígonos com corres diferenciadas e denominados Sítio 1, Sítio 2 e Sítio 3, apresentam áreas com tempo de cultivo diversificado, onde se pode observar áreas recém-implantadas, áreas com cultivos estabelecidos há quatro anos, áreas com período de cultivo entre quatro e dez anos, e por fim, áreas cultivadas com mais de vinte anos. Nas imagens foram identificadas ainda, informações típicas de solo exposto, água, nuvens e sombra das nuvens. Para determinação da produtividade primária bruta ou PPB, faz-se necessário a identificação previa de alguns parâmetros relevantes como temperatura e umidade relativa máxima e mínima, mês com maior valor médio do NDVI, além dos valores referentes à radiação fotossinteticamente ativa, temperatura média do ar do mês de máximo NDVI e a temperatura média do ar para o referido dia, apresentado na Tabela 2.

Tabela 2. Datas das imagens do OLI/TIRS, temperaturas do ar máxima - Tmax $\left({ }^{\circ} \mathrm{C}\right)$ e mínima Tmin $\left({ }^{\circ} \mathrm{C}\right)$, umidades relativas máxima - Urmax $(\%)$ e mínima - Urmin (\%), NDVI médio, radiação fotossinteticamente ativa - RFA $\left(\mathrm{MJ} \mathrm{m}^{-2} \mathrm{dia}^{-1}\right)$, valores de $\mathrm{T}_{1}$ e $\mathrm{T}_{2}$, referentes a temperatura média do ar para o mês com maior NDVI e temperatura média do ar diária.

\begin{tabular}{ccccccccc}
\hline Data & Tmax & Tmin & URmax & URmin & RFA & NDVI & T1 & T2 \\
\hline 03 de março & 35,3 & 21,2 & 79 & 28 & 11,45 & 0,827 & 0,974 & 0,956 \\
22 de maio & 34,7 & 21,1 & 75 & 25 & 10,98 & 0,864 & 0,974 & 0,951 \\
09 de julho & 32,8 & 20,2 & 77 & 28 & 9,96 & 0,860 & 0,974 & 0,956 \\
29 de outubro & 37,7 & 20,0 & 61 & 14 & 13,40 & 0,832 & 0,974 & 0,947 \\
14 de novembro & 36,8 & 24,1 & 67 & 24 & 11,07 & 0,807 & 0,974 & 0,893 \\
\hline
\end{tabular}


O vigor do cultivo e o nível de sanidade vegetal foram determinados com base nos valores do NDVI, sendo os mesmos indicativos da capacidade fotossintética e produção de biomassa das plantas. O mês de maio foi utilizado como referência, por apresentar o maior valor médio de NDVI $(0,864)$, como verificado na Figura 4. A radiação fotossinteticamente ativa (RFA) variou de 9 a 13 (MJ m dia $\left.^{-1}\right)$. Já o maior valor médio de RFA, observado no dia 29 de outubro $\left(13,4 \mathrm{MJ} \mathrm{m} \mathrm{mia}^{-1}\right)$ pode estar relacionado com a baixa cobertura de nuvens em decorrência do outono e início do verão, período caracterizado pela elevação das temperaturas e redução da umidade relativa do ar. Dentro dos cenários analisados, foram observadas variações nos valores do NDVI entre 0 e 1 para a vegetação nativa, pastagem, cultivo de sequeiro e irrigado (Figura 4). A PPB varia entre 5,0 e 7,5 (g C m$\left.{ }^{-2} \mathrm{dia}^{-1}\right)$ nos primeiros meses do ano, ficando abaixo de $2,5\left(\mathrm{~g} \mathrm{C} \mathrm{m}^{-2} \mathrm{dia}^{-1}\right)$ no segundo semestre (Figura 5). Segundo Almeida et al. (2018), esses valores são relativamente baixos quando comparados com outros ecossistemas como floresta $(8,5$ a 9,8 g C $\left.\mathrm{m}^{-2} \mathrm{dia}^{-1}\right)$, savana $\left(7,7 \mathrm{~g} \mathrm{C} \mathrm{m}^{-2} \mathrm{dia}^{-1}\right) \mathrm{e}$ pastagem $\left(7,0 \mathrm{~g} \mathrm{C} \mathrm{m}^{-2} \mathrm{dia}^{-1}\right)$. Entretanto, corroborando com os trabalhos de Silva et al. (2013), Teixeira et al. (2013) e Almeida et al. (2018), ao analisarem o aporte de biomassa para vegetação nativa e irrigada, tendo em vista, que vegetação nativa em ambas as pesquisas realizadas, trata-se de exemplares típicos da caatinga e/ou floresta de transição.

Apesar das adversidades climáticas, o cultivo irrigado da banana permanece estável e não apresenta redução acentuada nos valores da PPB, ressaltando a importância da precipitação e irrigação, para manutenção das atividades fisiológicas e produção de biomassa. Quando o cultivo da bananeira se encontra bem estabelecido, com plantas nos estágios de desenvolvimento e produção, a PPB varia de 7,5 a 10,0 ( $\left.\mathrm{g} \mathrm{C} \mathrm{m}^{-2} \mathrm{dia}^{-1}\right)$, como observado na Figura 5. Variações dos valores de PPB podem estar relacionadas com o tempo de implantação do pomar, haja vista, que podem ser encontradas áreas recentemente implantadas e outras com mais de vinte anos, corroborando com Teixeira et al. (2013), ao explicar que, a variabilidade dos valores de PPB em cultivos perenes pode está diretamente relacionado com o fornecimento hídrico, radiação incidente e a dinâmica de produção e colheita. 


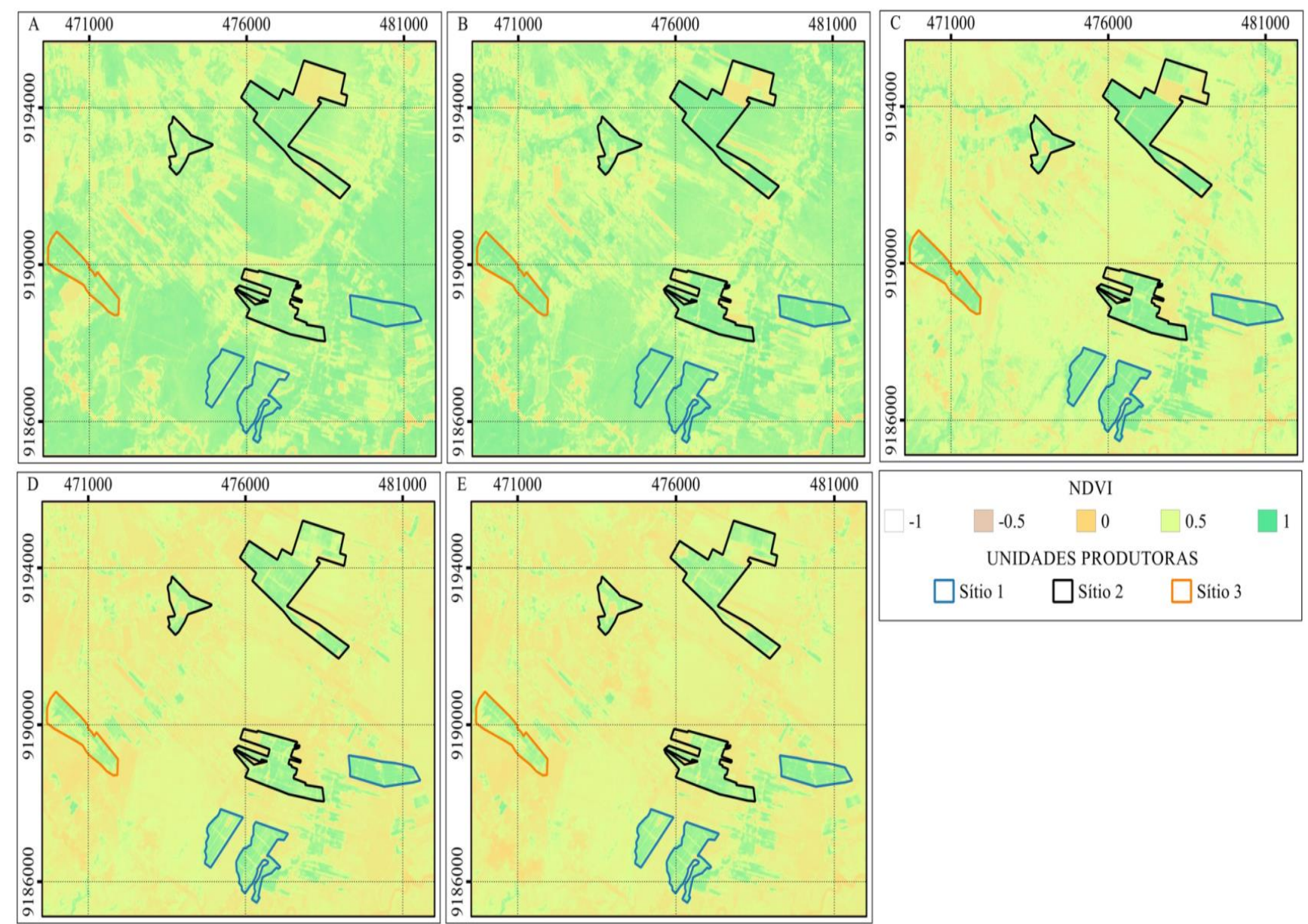

Figura 4. Espacialização geográfica dos valores de NDVI para a área em estudo no decorrer das datas: 3 de março (A), 22 de maio (B), 9 de julho (C), 29 de outubro (D) e 14 de novembro do ano de 2016.

Os valores do NDVI e PPB, obtidos para o cultivo irrigado da bananeira, encontram-se inseridos na faixa de valores observados por Silva et al. (2013), ao analisarem as referidas variáveis no perímetro irrigado de São Gonçalo - PB. Os autores salientam que, os valores de PPB próximos de $10 \mathrm{~g} \mathrm{C} \mathrm{m}^{-2} \mathrm{dia}^{-1}$ resultam da combinação entre elevada radiação solar, alto índice de vegetação, elevada fração evaporativa e fornecimento de demanda hídrica de forma adequada, reforçando a importância da irrigação para manutenção das atividades metabólicas, fixação de $\mathrm{CO}_{2}$ e aumento da produtividade do cultivo irrigado. Através da análise estatística, foi verificado que o cultivo irrigado pertencente à unidade produtora do Sítio 1 , de modo geral apresenta melhores médias em comparação com as demais unidades produtoras, tanto para a variável $\mathrm{PPB}$, como para o tempo de cultivo entre quatro e dez anos, como apresentado na tabela 2. 
Tabela 2. Análise estatística para Produtividade Primária Bruta - PPB ( $\left.\mathrm{g} \mathrm{C} \mathrm{m}^{-2} \mathrm{dia}^{-1}\right)$ para o cultivo irrigado da bananeira nas cincos datas avaliadas.

\begin{tabular}{|c|c|c|c|c|c|c|}
\hline \multirow{2}{*}{$\begin{array}{l}\text { Unidade } \\
\text { Produtora }\end{array}$} & \multicolumn{2}{|c|}{$\begin{array}{c}\text { Tempo de Cultivo } \\
4 \text { anos }\end{array}$} & \multicolumn{2}{|c|}{$\begin{array}{c}\text { Tempo de Cultivo } \\
4-10 \text { anos }\end{array}$} & \multicolumn{2}{|c|}{$\begin{array}{c}\text { Tempo de Cultivo } \\
10-20 \text { anos }\end{array}$} \\
\hline & & & \multicolumn{2}{|c|}{$\mathrm{PPB}\left(\mathrm{g} \mathrm{C} \mathrm{m}^{-2} \mathrm{dia}^{-1}\right)$} & & \\
\hline & & & \multicolumn{2}{|c|}{ 03/03/2016 } & & \\
\hline Sítio 1 & $7,09 \mathrm{aB}$ & $\pm 0,85$ & $7,42 \mathrm{aA}$ & $\pm 0,26$ & $6,95 \mathrm{aC}$ & $\pm 0,75$ \\
\hline Sítio 2 & $6,28 \mathrm{bC}$ & $\pm 1,17$ & $7,25 \mathrm{bA}$ & $\pm 0,29$ & $6,50 \mathrm{cB}$ & $\pm 0,73$ \\
\hline Sítio 3 & $5,78 \mathrm{cC}$ & $\pm 1,37$ & $7,29 \mathrm{aA}$ & $\pm 0,35$ & $6,83 \mathrm{bB}$ & $\pm 0,66$ \\
\hline \multicolumn{7}{|c|}{$22 / 05 / 2016$} \\
\hline Sítio 1 & $7,84 \mathrm{aB}$ & $\pm 0,41$ & $7,91 \mathrm{aA}$ & $\pm 0,21$ & $7,28 \mathrm{aC}$ & $\pm 0,61$ \\
\hline Sítio 2 & $7,52 \mathrm{bA}$ & $\pm 0,39$ & $7,62 \mathrm{bA}$ & $\pm 0,23$ & $7,00 \mathrm{cB}$ & $\pm 0,62$ \\
\hline Sítio 3 & $7,41 \mathrm{bB}$ & $\pm 0,49$ & $7,91 \mathrm{aA}$ & $\pm 0,23$ & $7,10 \mathrm{bA}$ & $\pm 0,57$ \\
\hline \multicolumn{7}{|c|}{$09 / 07 / 2016$} \\
\hline Sítio 1 & $6,98 \mathrm{aB}$ & $\pm 0,65$ & $7,38 \mathrm{aA}$ & $\pm 0,27$ & $6,53 \mathrm{aC}$ & $\pm 0,76$ \\
\hline Sítio 2 & $6,83 \mathrm{aA}$ & $\pm 0,56$ & $6,98 \mathrm{bA}$ & $\pm 0,33$ & $6,23 \mathrm{bB}$ & $\pm 0,77$ \\
\hline Sítio 3 & $6,14 \mathrm{bB}$ & $\pm 0,89$ & $7,31 \mathrm{aA}$ & $\pm 0,39$ & $6,12 \mathrm{cB}$ & $\pm 0,82$ \\
\hline \multicolumn{7}{|c|}{$29 / 10 / 2016$} \\
\hline Sítio 1 & $8,74 \mathrm{aA}$ & $\pm 0,64$ & $8,18 \mathrm{aB}$ & $\pm 0,75$ & $6,12 \mathrm{aC}$ & $\pm 1,47$ \\
\hline Sítio 2 & $8,09 \mathrm{bA}$ & $\pm 0,58$ & $7,61 \mathrm{cB}$ & $\pm 0,60$ & $5,72 \mathrm{cC}$ & $\pm 1,41$ \\
\hline Sítio 3 & $8,09 \mathrm{bA}$ & $\pm 1,01$ & $8,01 \mathrm{bA}$ & $\pm 0,71$ & $5,90 \mathrm{bB}$ & $\pm 1,57$ \\
\hline \multicolumn{7}{|c|}{$14 / 11 / 2016$} \\
\hline Sítio 1 & $6,31 \mathrm{aA}$ & $\pm 0,57$ & $6,01 \mathrm{aB}$ & $\pm 0,52$ & $4,71 \mathrm{aC}$ & $\pm 1,06$ \\
\hline Sítio 2 & $5,62 \mathrm{bA}$ & $\pm 0,67$ & $5,53 \mathrm{cA}$ & $\pm 0,39$ & $4,18 \mathrm{cB}$ & $\pm 0,93$ \\
\hline Sítio 3 & $5,50 \mathrm{bB}$ & $\pm 0,88$ & $5,87 \mathrm{bA}$ & $\pm 0,48$ & $4,39 \mathrm{bC}$ & $\pm 1,09$ \\
\hline
\end{tabular}

Médias seguidas pelas mesmas letras maiúsculas na linha e minúsculas na coluna não diferem pelo teste de Tukey a $5 \%$ de probabilidade. 

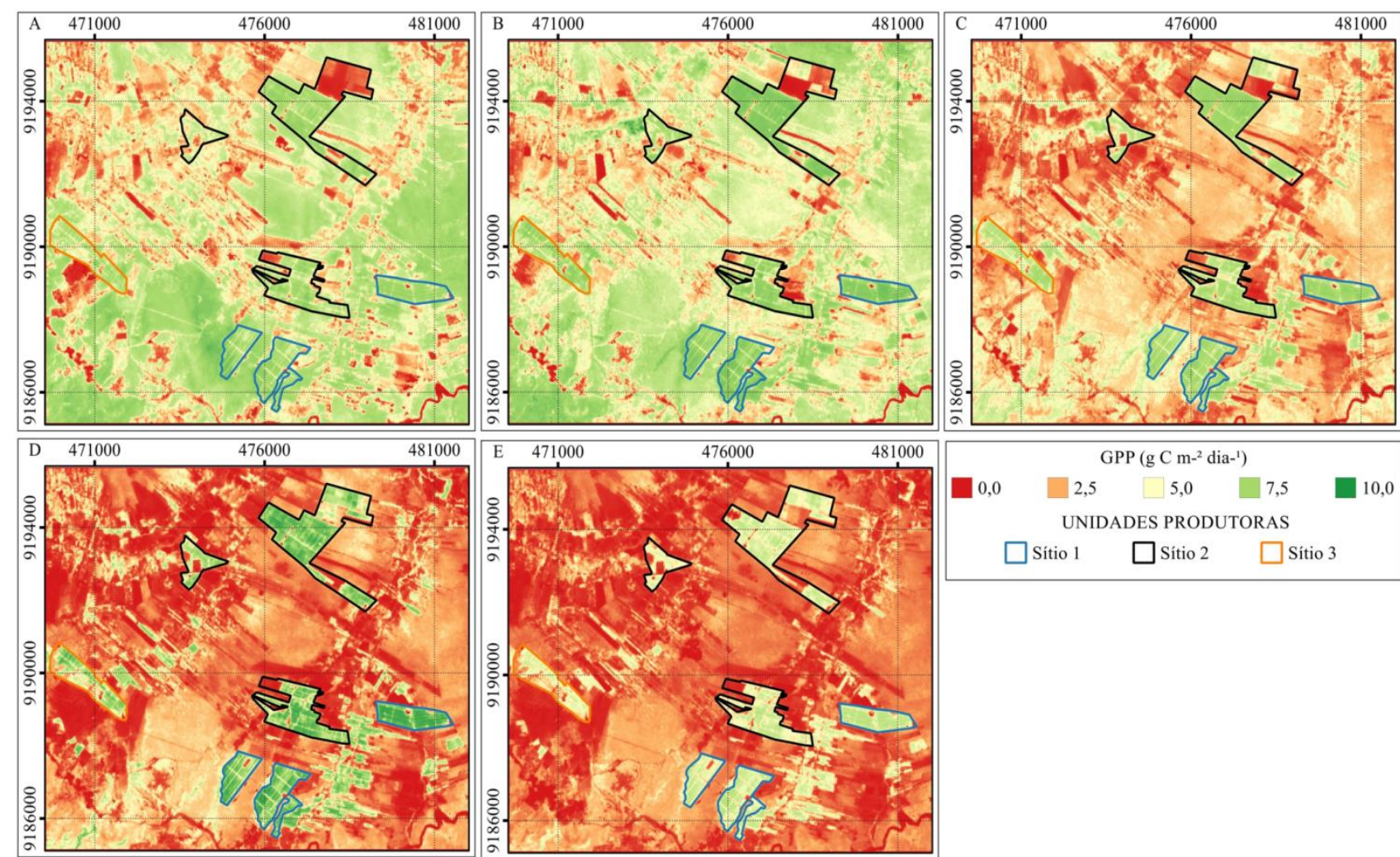

Figura 5. Espacialização geográfica dos valores da PPB $\left(\mathrm{g} \mathrm{C} \mathrm{m}^{-2} \mathrm{dia}^{-1}\right)$ para a área em estudo no decorrer das datas: 3 de março (A), 22 de maio (B), 9 de julho (C), 29 de outubro (D) e 14 de novembro do ano de 2016.

As variações observadas para a variável PPB, verificadas nas unidades produtoras, estão relacionadas com o período em análise, uma vez que, no início do ano de 2016 observam-se temperaturas amenas, maiores precipitações e umidade do solo (Figura 2).

O compilado destes fatores resulta em maiores taxas de evapotranspiração, produção de biomassa e melhor eficiência do uso da água de irrigação, empregada no cultivo da banana.

De acordo com a unidade produtora do Sítio 1 , a produtividade média obtida durante $o$ período entre $1^{\circ}$ de março e 31 de novembro de 2016, foi de aproximadamente 25,09 $\mathrm{t} \mathrm{ha}^{-1}$ de frutos, valor considerado satisfatório, uma vez que se encontra inserido no intervalo recomendado por Silva et al. (2013). No Sítio 2, verificou-se produtividade média de aproximadamente $35,00 \mathrm{t} \mathrm{ha}^{-1}$ de frutos, o que diverge de Silva et al. (2013). Porém, este valor pode estar relacionado ao fato de que a maior parte da área cultivada se encontra com tempo de cultivo de quatro a dez anos. E por fim, a unidade produtora do Sítio 3, afirmou ter obtido produtividade média de apenas $16 \mathrm{t}$ $\mathrm{ha}^{-1}$, valor considerado baixo por Silva et al.
(2013), Santos et al. (2014) e Souza et al. (2017).

\section{CONCLUSÕES}

O cultivo irrigado nas unidades produtoras apresenta melhor eficiência do uso da água, em comparação com a vegetação nativa, pastagem e cultivo de sequeiro, uma vez que a produtividade primaria bruta foi superior a $8 \mathrm{~g} \mathrm{C} \mathrm{m}^{-2} \mathrm{dia}^{-1}$ durante o período de maior demanda hídrica. Provavelmente, o manejo adotado no cultivo da bananeira influenciou para que a unidade produtora do Sítio 1 obtivesse melhores resultados e maior produtividade. No que se refere aos tempos de cultivo, verificou-se que áreas cultivadas entre quatro e dez anos foram superiores em relação às demais.

\section{REFERÊNCIAS BIBLIOGRÁFICAS}

ALLEN, R. G.; TASUMI, M.; TREZZA, R.; KJAERSGAARD, J. H. Mapping evapotranspiration at high resolution, application manual for Landsat satellite 


\section{ESTIMATIVA DA PRODUTIVIDADE PRIMÁRIA BRUTA DA VEGETAÇÃO NATIVA E CULTIVO IRRIGADO ATRAVÉS DE SENSORIAMENTO REMOTO}

imagery. Version 2.0.7. University of Idaho, Kimberly, p. 248, 2010.

ALMEIDA, C. T.; DELGADO, R. C.; GALVÃO, L. S.; ARAGÃO, E. O. C. L.; RAMOS, M. C. Improvements of the MODIS Gross Primary Productivity model based on a comprehensive uncertainty assessment over the Brazilian Amazonia. ISPRS Journal of Photogrammetry and Remote Sensing, $n$. July, p. 1-16, 2018. DOI: 10.1016/j.isprsjprs.2018.07.016.

BASTIAANSSEN, W. G. M. BRITO, R. A. L.; BOS, M. G.; SOUZA, R. A.; CAVALCANTI, E. B.; BAKKER, M. M. Low cost satellite data for monthly irrigation performance monitoring: benchmarks from Nilo Coelho, Brazil. Irrigation and Drainage Systems, v. 15, n. 1, p. 53-79, 2001. DOI: 10.1023/A:1017967021198.

BASTIAANSSEN, W. G; ALI, S. A new crop yield forecasting model based on satellite measurements applied across the Indus Basin. Agriculture, Ecosystems and Environment, v. 94, n. 3, p. 321-340, 2003. DOI: 10.1016/S0167-8809(02)00034-8.

BEER, C.; REICHSTEIN, M.; TOMELLERI, E.; CIAIS, P.; JUNG, M.; CARVALHAIS, N.; RÖDENBECK, C.; ARAIN, M. A.; BALDOCCHI, D.; BONAN, G. B.; BONDEAU, A.; CESCATTI, A.; LASSLOP, G.; LINDROTH, A.; LOMAS, M.; LUYSSAERT, S.; MARGOLIS, H.; OLESON, K. W.; ROUPSARD, O.; VEENENDAAL, E.; VIOVY, N.; WILLIAMS， C.; WOODWARD， F. I.; PAPALE, D. Terrestrial gross carbon dioxide uptake: global distribution and covariation with climate. Science, v. 329, n. 5993, p. 834838, 2010. DOI: 10.1126/science. 1184984 .

BRANDÃO, Z. N.; SOUZA, J. Y. B.; BARBOSA, M. P.; ZONTA, J. H.; BEZERRA, J. R. C. Zoneamento de áreas de plantio e mapeamento de APPS e RLS usando imagens ALOS. In: Simpósio Brasileiro de
Ciências Geodésias e Tecnologias da Geoinformação, 4., 2012, Recife. Anais... Recife: UFPE, 2012. p. 001-006.

CABRAL, O. M. R.; GASH, J. H. C.; ROCHA, H. R.; MARSDEN, C.; LIGO, M. A. V.; FREITAS, H. C.; TATSCH, J. D.; GOMES, E. Fluxes of $\mathrm{CO} 2$ above a plantation of Eucalyptus in southeast Brazil. Agricultural and Forest Meteorology, v. 151, p. 49-59, 2011.

COSTA, F. S.; SUASSUNA, J. F.; MELO, A. S.; BRITO, M. E. B.; MESQUITA, E. F. Crescimento, produtividade e eficiência no uso da água em ba-naneira irrigada no semiárido paraibano. Caatinga, v. 25, n. 4, p. 26-33, 2012.

DOUGHTY, R.; XIAO, X.; WU, X.; ZHANG, Y.; BAJGAIN, R.; ZHOU, Y. QIN, Y.; ZOU, Z.; McCARTHY, H. R.; FRIEDMAN, J. R.; WAGLE, P.; BASARA, J. B. STEINER, J. L. Responses of gross primary production of grasslands and croplands under drought, pluvial, and irrigation conditions during 20102016, Oklahoma, USA. Agricultural Water Management, v. 204, p. 47-59, 2018. DOI: 10.1016/j.agwat.2018.04.001.

FAO Stat. FAO Stat accessed vide http://www.fao.org/faostat/en/\#data/QC on 28/09/2019.

FERREIRA, D. F. Sisvar: a computer statistical analysis system. Ciência e Agrotecnologia, v. 35, n.6, p. 1039-1042, 2011.

FIELD, C. B.; RANDERSON, J. T.; MALMSTROM, C. M. Global net primary production: combining ecology and remote sensing. Remote Sensing of Environment, v. 51, p. 74-88, 1995. DOI: 10.1016/00344257(94)00066-V.

IBGE. Sistema IBGE de Recuperação Automática - SIDRA. Produção Agrícola Municipal. 2017. Disponível em: 
www.sidra.ibge.gov.br. Acesso em: 24 fev. 2019.

LIMA, M. T. V.; MEIRELES, A. C. M.; OLIVEIRA, C. W.; NASCIMENTO, M. T. B. Koppen-Geiger and Thornthwaite climatic classification for the metropolitan region of the Cariri, Ceará. GEAMA, v. 3, n. 3, p. 136143, 2017.

MEDEIROS, R. M.; SILVA, J. A. S.; SILVA, A. O. MATOS, R. M.; BALBINO, D. P. Balanço hídrico climatológico e classificação climática para a área produtora da banana do município de Barbalha, CE. Revista Brasileira de Agricultura Irrigada, v. 7, n. 4, p. 258-268, 2013. DOI: 10.7127/rbai.v7n400018.

MONTEITH, J. L. Solar radiation and productivity in tropical ecosystems. Journal of Applied Ecology, v. 9, p. 747-766, 1972. DOI:10.2307/2401901.

ROBINSON, J. C.; SAÚCO, G. V. Bananas and plantains. 2nd ed. Oxford: CAB International, 2010, p. 311.

SANTOS, C. N.; OLIVEIRA, I. C. P.; DONATO, S. L. R.; ARANTES, A. M.; ROSA, R. C. C. Área e índice de área foliar de bananeiras 'prata-anã' e 'BRS platina' submetidas à adubação orgânica. CONGRESSO BRASILEIRO DE FRUTICULTURA. 22., Cuiabá. Anais... Cuiabá: 2014. p. 720-724.
SHARMA, T.; KURZ W. A.; STINSON, G.; PELLATT, M. G.; LI, Q. 100-year conservation experiment: impacts on forest carbon stocks and fluxes. Forest Ecology and Management, v. 310, p. 242-255, 2013, DOI: 10.1016/j.foreco.2013.06.048.

SILVA, B. B.; GALVÍNCIO, J. D.; MARIA, S.; MONTENEGRO, G. L.; MACHADO, C. C. C.; MARIA, L.; OLIVEIRA, M.; MOURA, M. S. B. Determinação por sensoriamento remoto da produtividade primária bruta do perímetro irrigado São Gonçalo-PB. Revista Brasileira de Meteorologia, v. 28, n. 1, p. 5764, 2013.

SOUZA, G.; ALENCAR, G. S. S.; ALENCAR, F. H. H. Análise socioambiental da produção de banana no município de Cariús-CE, Brasil. Ciência e Sustentabilidade, p. 119-144, 2017.

TEIXEIRA, A. H. C.; WARREN-SCHERER, M.; HERNANDEZ, F. B. T.; ANDRADE, R. G. Large-scale water productivity assessments with MODIS images in a changing semi-arid environment: a brazilian case study. Remote Sensing. v. 5, p. 5783-5804, 2013.

ZHONG, L.; HU, L.; YU, L.; GONG, P.; BIGING, G. S. Automated mapping of soybean and corn using phenology Liheng. ISPRS Journal of Photogrammetry and Remote Sensing, v. 119, p. 151-167, 2016. 